\title{
Design an Equivalent Left Ventricular Assist Device for Medical Equipment Labs
}

\author{
Nazeeh N. N. ${ }^{1 \odot}$, Aljobouri H. K. ${ }^{1 * \odot}$, Odai M. ${ }^{1}$
}

\begin{abstract}
LVAD is a mechanical pump supporting a weak heart function and blood flow. Sometimes, the heart may not recover fast enough to take over the pumping action immediately after surgery, in such patients a temporary support device has been employed to maintain the pumping action until the patient's own heart recovers. This device can be considered as a temporary alternative before the process of artificial heart transplantation. In this work, a new equivalent Left Ventricular Assist Device (LVAD) is designed and implemented as a simple circuit for medical equipment labs. The presented LVDA consists of a mechanical motor, tubes, a power source, and microcontroller. The output results show the range of readings near the percentage ranges of the left ventricular pumping of the human adult. This work is significant for the biomedical equipment's lab. The researcher can deal with the function of the important medical devices which are artificial that can record different readings.
\end{abstract}

Citation: Nazeeh N. N, Aljobouri H. K, Odai M. Design an Equivalent Left Ventricular Assist Device for Medical Equipment Labs. J Biomed Phys Eng. 2019;9(4):495-500. https://doi.org/10.31661/jbpe.v0i0.1171

\section{Keywords}

Durable Medical Equipment • Equipment Design • Heart Ventricles

\section{Introduction}

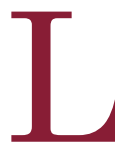
eft Ventricular Assist Device (LVAD) is considered for long-term implantation, it can create flows up to 10 liters per minute (lpm) free to the left ventricular flow. Currently, there is no recognized preoperative seer of right ventricular failure in patients needing LVAD implantation. The LVAD internal surfaces consist of the thin-walled duct, rotor, outlet stator, and inlet stator. They have fine refined titanium surfaces. The patient may be fully moveable for long periods, needing only a wearable system controller and portable batteries. The volume of flow produced by the Left Ventricular Assist Device (LVAD) is defined by the differential pressure that exists across the pump and the speed of the rotor rotation. For a definite speed, flow differs inversely with pressure. Thus, when the flow decreases, the pump differential pressure will increase $[1,2]$.

In 1966, the first of a left ventricular assist device implantation was successfully conducted by Dr. Michael E. DeBakey to a woman who was 37 years old. An external paracorporeal system was arranged for mechanical sustenance during 10 days next to the surgery [3]. While in 1988, the first implantation of an artificial LVAD for a long-term time was successfully introduced by Dr. William F. Bernhard of Boston Chil-
${ }^{1}$ Biomedical Engineering Department, College of Engineering, Al-Nahrain University, Baghdad, IRAQ

Corresponding author: H. K. Aljobouri Biomedical Engineering Department, College of Engineering, Al-Nahrain University, Baghdad, IRAQ

E-mail:hadeel_bme77@ yahoo.com

Received: 24 April 2019 Accepted: 25 June 2019 
dren's Hospital Medical Center and Thermedics, Inc of Woburn, MA. He advanced HeartMate which is an electronically controlled assist device under a National Institutes of Health (NIH) research contract [4]. In 1994, the initial VADs simulated the heart via a "pulsatile" action. The blood is consecutively drawn from the left ventricle into the pump and then pushed into the aorta. This type of device involved the HeartMate IP LVAS in the US; it was permitted for practice by the Food and Drug Administration (FDA). These systems are generally stated as the first VADs generation. The further research recently carried out has focused on continuous flow pumps. It can be approximately regarded as either axial flow impulse driven or centrifugal pumps. These pumps have the improvement of more easiness resulting in better reliability and reduced size. These systems are stated as second VADs generation [5].

The Left ventricular assist device is an important device used for keeping the patients alive until transplantation and the heart "rest" after trauma. In infrequent subjects, the heart can be recovered from its normal ability by giving it away to rest (Figure 1). Furthermore, it supports patients who cannot have a heart transplant by performance exercise and also improves or maintains other organs. LVAD returns blood flow to a patient who has a weak heart due to various heart diseases. This supports relieve several signs, such as the frequently tired or short of breath [6].

A simple block diagram of the proposed LVDA system is shown in Figure 2. It consists of:

- Pump: a mechanical motor to pump the fluid from the left ventricular

- Flow sensor: sensed the water flow rate

- Microcontroller: programmable controller (Arduino UNO) programming by the computer running a custom instruction by using Arduino (IDE) software

- Power source: supply a pump and microcontroller

- Tubes: to move blood out of the heart and blood vessels

- Liquid crystal display (LCD): show flow rate output results

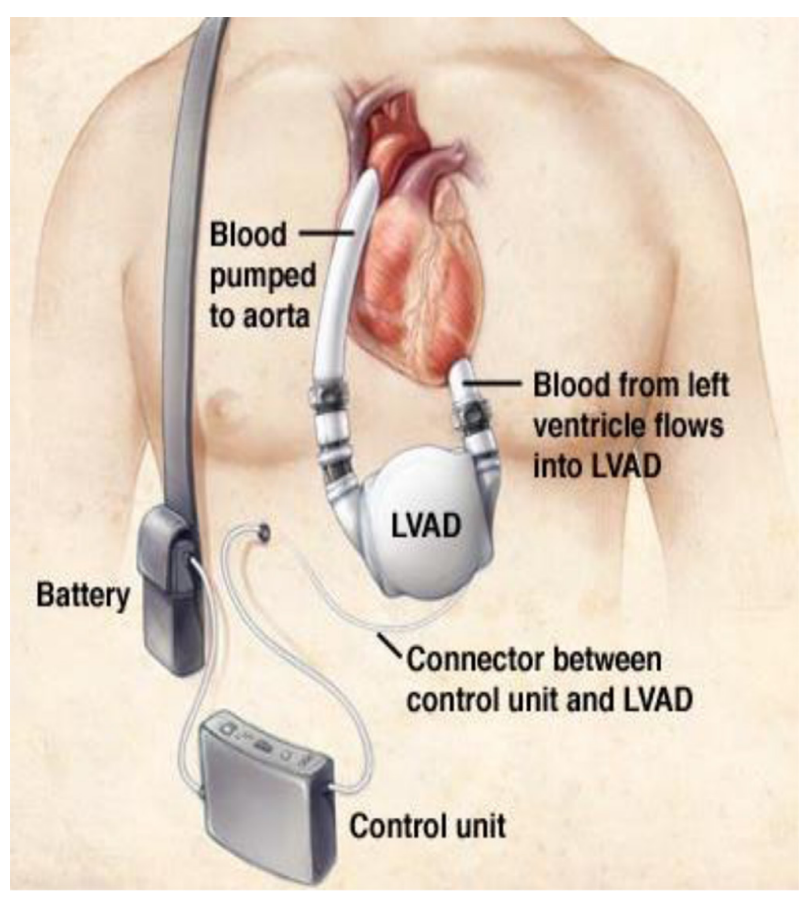

Figure 1: Left ventricular assist device (LVAD) [7]. 


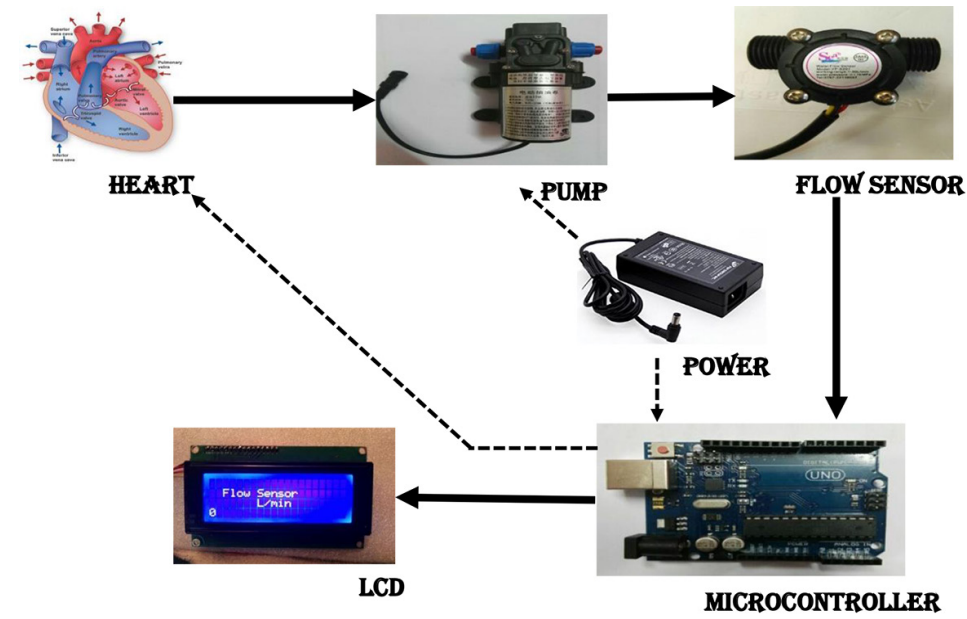

Figure 2: A simplified block diagram of the LVDA.

The rest of this article is organized as follows. The construction design and the proposed LVAD are described in Section 2. All the experimental output results are presented in Section 3. Finally, the conclusions and discussion are presented in Section 4.

\section{Material and Methods}

The main components of the proposed LVAD are presented as follows:

a) A motor pump which is used to push the blood from left ventricular. Water is used instead of blood in the presented mechanical design.

b) Flow sensor which is used for the flow rate sensing [8].

c) Microcontroller: one programmable controller (Arduino Uno) is used. It is programming by computer and running a custom instruction by using Arduino (IDE) software that control both of sensor and Liquid crystal.

d) Small breadboard.

e) A container that represented left ventricular.

f) Elastic pipe.

g) Transformer which is used as a power supply for both Arduino and pump.

h) Liquid crystal (LCD 20*4) to display information taken by the sensor [9].
The flow sensor sits in the water line using a pinwheel sensor to a degree the amount of moving liquid through it. The sensors are stably built and run a digital pulse whenever a volume of water comes through the pipe. The flow sensor was controlled by the microcontroller; therefore, the output can simply be connected for monitoring the amount of used water. The microcontroller has the ability to compute how much water residual is in a container.

The used microcontroller is constructed on the ATmega328 [10]. It has 14 digital input/ output pins. The programmable controller Arduino UNO was used which has many features with respect to other types of the microcontroller. It can be supplied with electric power easily with no high voltage to activate. Also, Arduino UNO is small in size so that it can be handled easily. Furthermore, it is available and not costly. It can be possible to work with different types of electronic components and even with sensors. Arduino UNO can be programmed easily, using Arduino IDE software that is easy to install on the computer and able to change and fixe the instruction used. Figure 3 shows the connections between Arduino, LCD display and flow sensor.

The motor pump used in this work has no 


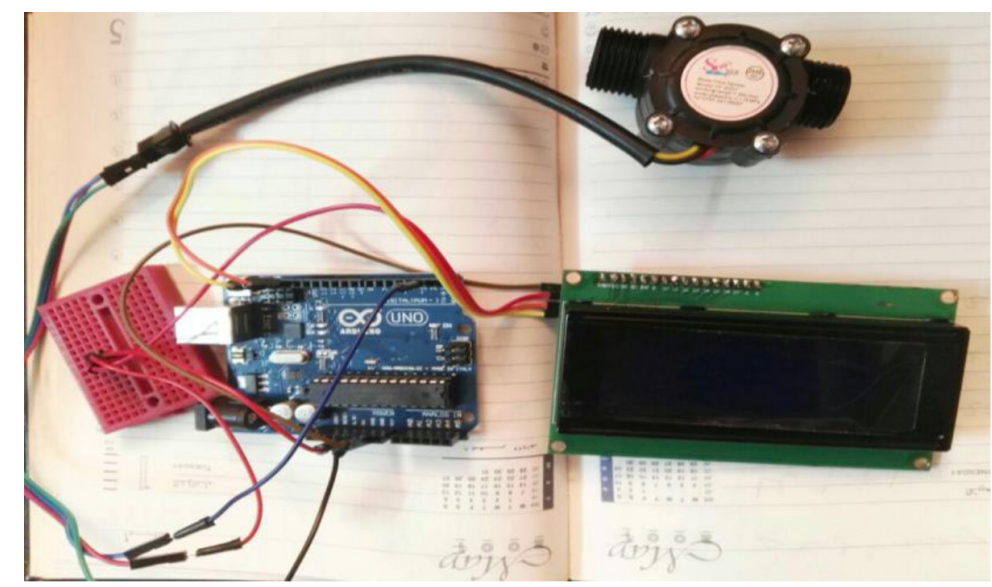

Figure 3: LCD and flow sensor connection to Arduino.

strict requirement on the size of the water outlet. When the water outlet hole is too small or blocked, the motor does not stop pumping. The pump must be used in a dry condition; thus it can't be submerged in water.

The elastic pipe used in the design is a tube which has a length of 2.5 meters. This tube is considered as the length of the blood cycle of the human body and has a diameter of $1 \mathrm{~cm}$.

The container represents the left ventricle of the human, and its capacity is approximately $200 \mathrm{ml}$. The container was chosen as it was close to the volume of the left ventricle of the human as its size ranged from $142 \mathrm{~mL}( \pm 30$ $\mathrm{mL})$ [11]. The final implemented design is shown in Figure 4.

\section{Results}

There are different experiments performed for the proposed and implemented LVAD design. To test the flow sensor working, two experiments have been implemented. In the first experiment, the sensor was placed above the container, and the water poured on it without venting the container. The value of output results are stabilized at $0 \mathrm{~L} / \mathrm{m}$, as shown in Figure 5. Figure 6 shows the LCD which displayed " 0 " value for the flow sensor in $(\mathrm{L} / \mathrm{m})$ when the container is empty. In the second experiment, the container was ventilated. The value of output results increased because of the lower internal pressure, as shown in Figure 7.

When the water is added with different val-

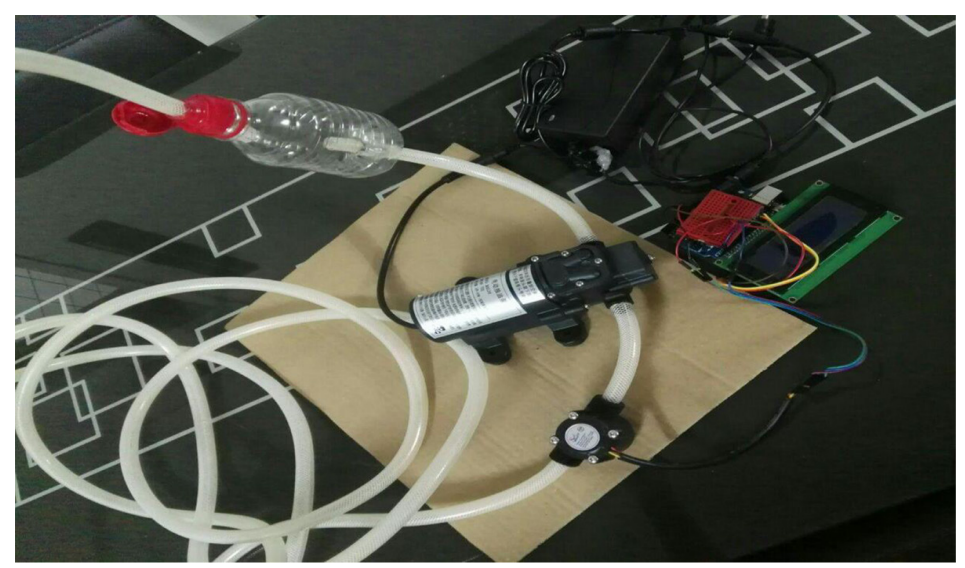

Figure 4: The final design implemented. 
ues, including 120, 150 and $200 \mathrm{ml}$ to the container, the results has been grouped as shown in Table 1.

\section{Discussion}

The main purpose of the LVDA is to support patients with weak heart function and blood

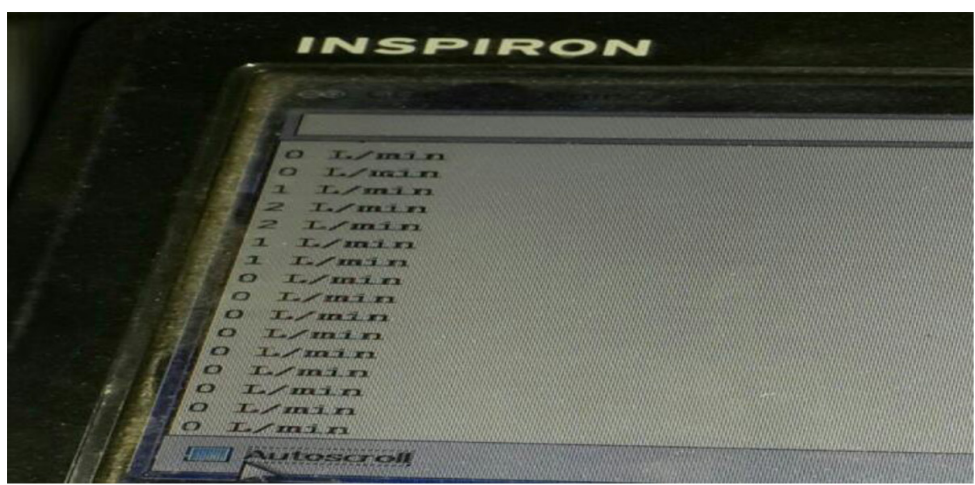

Figure 5: The results when the flow sensor above the container.

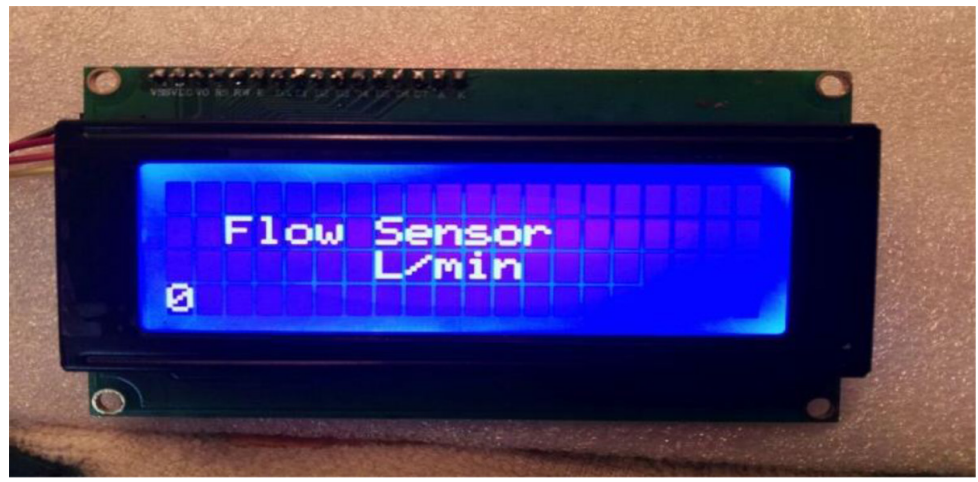

Figure 6: The output result when the container is empty.

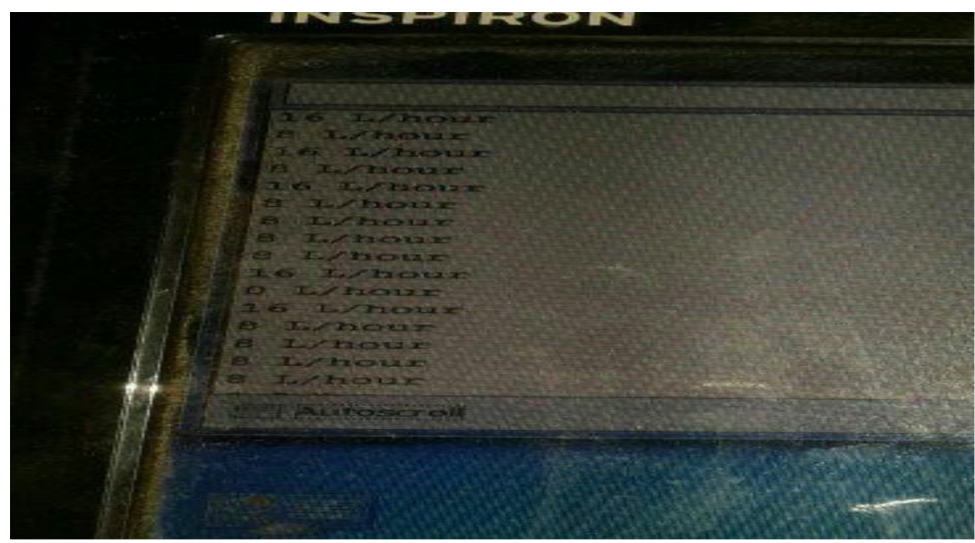

Figure 7: The results when the flow sensor is ventilated. 
Table 1: Show the flow rate on the LCD.

\begin{tabular}{cc} 
Amount of water in (ml) & Flow in (L/min) \\
\hline \multirow{2}{*}{120} & 6 \\
\cline { 2 - 2 } 120 & 7 \\
\hline \multirow{2}{*}{200} & 7 \\
\hline \multirow{2}{*}{150} & 8 \\
\hline
\end{tabular}

flow. In this work, the designed LVDA system is included: motor, tubes, power source, microcontroller, and flow sensor. A mechanical motor is used to pump the fluid from the left ventricular, while the tubes used to transfer blood out of the heart and to blood vessels. A programmable controller Arduino UNO is used as a microcontroller, and all the output results are displayed on the LCD. The percentage of left ventricular pumping of the adults in the normal state ranges from (8-5) liters per minute. The output results of the proposed device are approximately the same as those of the normal person, and the reading ranges were from (8-6) liters per minute.

\section{Conclusion}

The presented work is crucial important for the medical and biomedical equipment's lab, thus the student can be familiar with the working in this type of important artificial medical devices. The researcher can deal with the device and record different readings. This work is simple and no costly. For future work, the presented design can be modified by using bubble traps. Bubble traps can rapidly clear bubbles to ensure consistent and reliable system fluids; therefore, the problem causing bubbles will be solved. Another suggestion for a new design is using a solenoid valve. This valve is electromechanically operated in the inflow of the container, and it has the ability to calculate the filling time of the left ventricular.

\section{Conflict of Interest}

\section{None}

\section{References}

1. HeartMate II TM Left Ventricular Assist Device [Internet]. Heartmate II TM left ventricular assist system. [cited 25 March 2019]. Available from: https://www.heartmate.com/SJH/media/Emerge_ SJH/pdf/22774_SJM-HM-0317-0061a_HeartMateII-Product-Brochure.pdf

2. Miller GE. Artificial organs. Williston: Morgan \& Claypool Publishers; 2006. P. 66.

3. Kirklin JK, Naftel DC. Mechanical circulatory support: registering a therapy in evolution. Circ Heart Fail. 2008;1:200-5. doi: 10.1161/CIRCHEARTFAILURE.108.782599. PubMed PMID: 19808290; PubMed Central PMCID: PMC3437761.

4. Shah $A$ [Internet]. Evaluation of the HeartWare ${ }^{\circ}$ LVAD System for the Treatment of Advanced Heart Failure. c2009. [cited 25 March 2019]. Available from: http://www.hopkinsmedicine.org/heart_vascular_institute/clinical_trials/mechanical_circulatory_support/heartware_Ivad_system_heart_failure. html

5. Popov AF, Hosseini MT, Zych B, Simon AR, Bahrami T. HeartWare left ventricular assist device implantation through bilateral anterior thoracotomy. Ann Thorac Surg. 2012;93:674-6. doi: 10.1016/j.athoracsur.2011.09.055.PubMed PMID: 22269746.

6. Baum D. No Pulse: How Doctors Reinvented The Human Heart. Popular Science. 2012:36.

7. Mayo Clinic [Internet]. Left ventricular assist device (LVAD). [cited 25 March 2019]. Available from: https://www.mayoclinic.org/tests-procedures/ventricular-assist-device/multimedia/left-ventricularassist-device/img-20006714

8. Sensors \& Sensor Modules [Internet]. YF-S201 Water Flow Sensor. [cited 25 March 2019]. Available from: https://files.amperka.ru/store-media/ products/water-flow-sensor/media/YF-S201.pdf

9. Castellano JA. Liquid gold: the story of liquid crystal displays and the creation of an industry. London: World Scientific; 2005. P. 302.

10. Electronic Componentd Datasheet Search [Internet]. A000066 Datasheet, PDF. [cited 25 March 2019]. Available from: http://www.alldatasheet. com/view.jsp?Searchword=A000066\&gclid=Cj wKCAjw-OHkBRBkEiwAoOZql0B-b9pdRHaDAg2djsPzRkaRXgiPYf5ljT2Vq1v5ZjP48FI9Dgi_zBoCMp4QAvD_BwE

11. Maceira AM, Prasad SK, Khan M, Pennell DJ. Normalized left ventricular systolic and diastolic function by steady state free precession cardiovascular magnetic resonance. J Cardiovasc Magn Reson. 2006;8:417-26. doi: 10.1080/10976640600572889. PubMed PMID: 16755827. 